\title{
Modelling of bionic arm
}

\author{
Amartya Ganguly
}

Department of Biomedical Engineering, JIS College of Engineering; Block 'A', Phase III, Kalyani, Nadia, India. Email: ganguly.amartya@yahoo.in

Received 11 December 2009; revised 25 December 2009; accepted 29 December 2009.

\begin{abstract}
The bionic arm is a prosthesis which will allow the amputees to control it with the help of their own brain instead of depending upon the mechanical functions of the artificial limbs which are at present available in the market. A complex design of control systems is embedded in the bionic arm which will receive and analyze the signals from the brain and convert the electrical energy to mechanical energy, making the bionic arm move.
\end{abstract}

Keywords: Neural Network; Bionic, Amputation; Upper Limb

\section{INTRODUCTION}

Bionic arm is a revolutionary idea for amputees across the globe. This is as close as we can get to our natural limb. The fundamental point is to make the arm move with our brain unlike previous prosthetic upper limbs. In the case of bionic arm we take the nerve conduction signals from the brain and amplify it so that we can register the signal and convert that electrical signal into mechanical energy so as to move the mechanical device i.e. the arm. Prosthesis is being used and constantly being perfected to suit human needs. Various types of prosthesis have been made to suit many actions but not all. But the bionic arm will be able to perform all kinds of movements of the human upper limb even the most difficult actions like unscrewing the cap of bottle or picking up a coin from the ground. This arm will also be able to judge the correct pressure required for any movement.

\section{METHODS}

The electrodes placed near the chest region will detect the strongest of the nerve impulses from the brain which is then fed to the biopotential amplifier to amplify the signals. The amplified signals are then routed to the transducer to convert this electrical energy to mechanical energy enabling the bionic arm to move as per the strength of the signal. This can be further explained by mathematics as given below.

This here is a mathematical model in order to simulate the function of the brain. For this purpose the NARMA L2 Controller has been used. To put it more generally neural networks have been used [1].

\subsection{Model Components}

The Figure 1 above shows the arrangement of the circuit components of the Model of Bionic Arm. [2,3]The uniform random signal goes to the brain that is the Narma L2 Controller which goes to the subsystem of bionic arm which consists of the biopotential amplifier and the transducer, Figure 2 shown below. The graphical result is viewed in the scope.

\subsection{Narma L2 Controller}

[4]The neurocontroller described in this section is referred to by two different names: feedback linearization control and NARMA-L2 control. It is referred to as feedback linearization when the plant model has a particular form (companion form). It is referred to as NARMA-L2 control when the plant model can be approximated by the same form. The central idea of this type of control is to transform nonlinear system dynamics into linear dynamics by canceling the nonlinearities. Figure 3 represents the training to identify the Narma L2 controller. This section begins by presenting the companion form system model and demonstrating how you can use a neural network to identify this model. Then it describes how the identified neural network

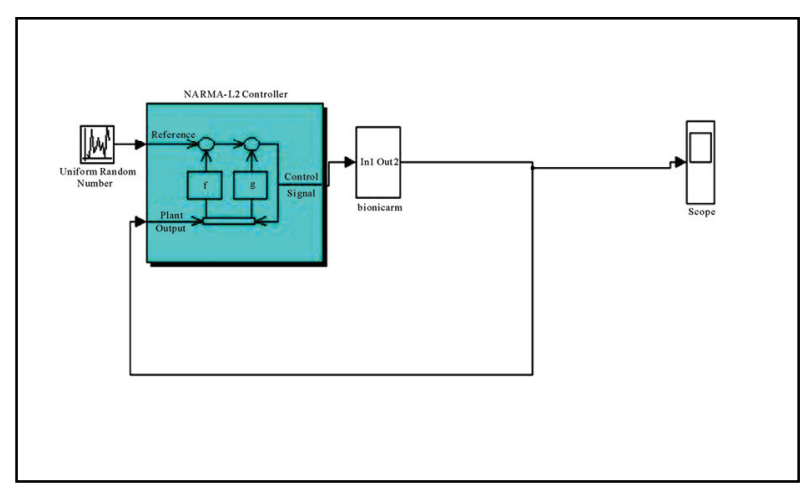

Figure 1. Block diagram of bionic arm. [5] 


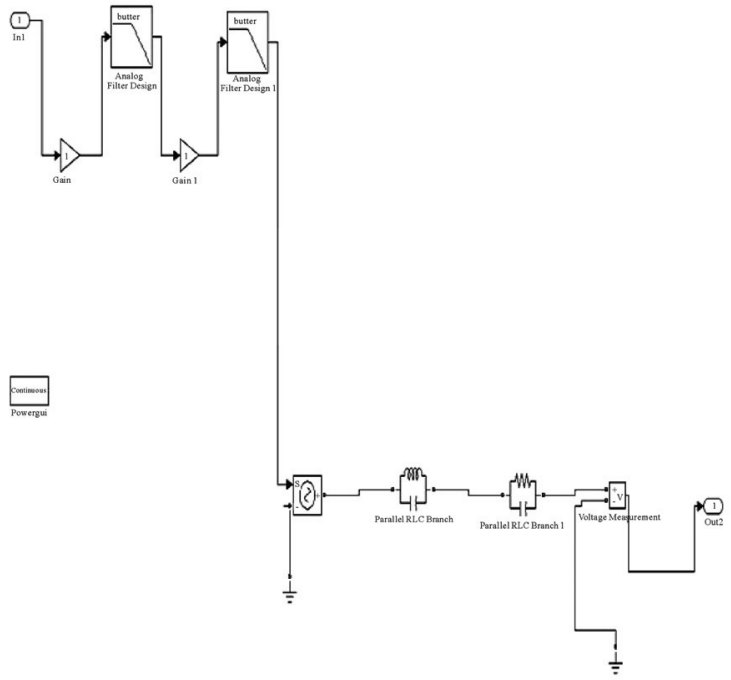

Figure 2. Circuit Diagram of Biopotential Amplifier and Transducer. [5]

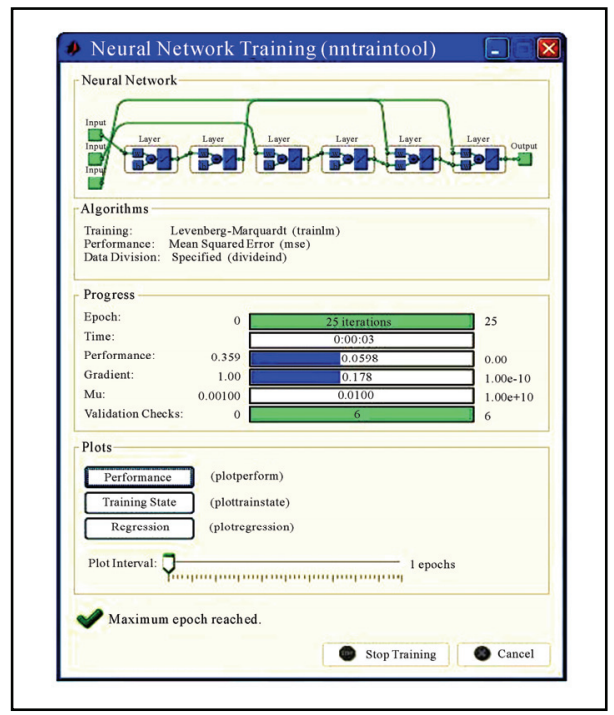

Figure 3. Neural Network Training to identify the Narma L2 controller. [5]

model can be used to develop a controller. This is followed by a demonstration of how to use the NARMAL2 Control block, which is contained in the Neural Network Toolbox ${ }^{\mathrm{TM}}$ block set.

\section{IDENTIFICATION OF NARMA-L2 MODEL}

As with model predictive control, the first step in using feedback linearization (or NARMA-L2) control is to identify the system to be controlled. You train a neural network to represent the forward dynamics of the system. The first step is to choose a model structure to use. One standard model that is used to represent general dis- crete-time nonlinear systems is the nonlinear autoregressive-moving average (NARMA) model. The notation MA $(q)$ refers to the moving average model of order $q$ :

$$
X_{t}=c+\varepsilon_{t}+\sum_{i=1}^{p} \varphi_{i} X_{t-i}+\sum_{i=1}^{q} \theta_{i} \varepsilon_{t-i}
$$

Where the $\theta_{1}, \ldots, \theta_{q}$ are the parameters of the model, $\mu$ is the expectation of $X_{t}$ (often assumed to equal 0 ), and the, $\varepsilon_{t}, \varepsilon_{t-1}, \ldots$ are again, white noise error terms. The moving average model is essentially a finite impulse response filter with some additional interpretation placed on it. This is the identification procedure used for the NN Predictive Controller.

The only problem with using this controller is that if you want to train a neural network to create the function $\mathrm{G}$ to minimize mean square error, you need to use dynamic backpropagation ([NaPa91] or [HaJe99]). This can be quite slow. One solution, proposed by Narendra and Mukhopadhyay [NaMu97], is to use approximate models to represent the system. The controller used in this section is based on the NARMA-L2 approximate model.

\section{RESULTS}

Since the brain receives various signals to perform a wide range of functions pertaining to different systems of the human body any unknown system would require training of the brain, in this case the NARMA L2 Controller. We have to train it so that it can identify which signals are exclusively for the movement of the upper limb. The Figures 4, 5, 6 and 7 show us the various stages the controller has to go through before we can ascertain that the signals received are for the movements of the upper extremity. Here, random uniform signals have been taken as input data similar to the brain which constantly receives and emits various types of signals for different functions. The neural network will be able to

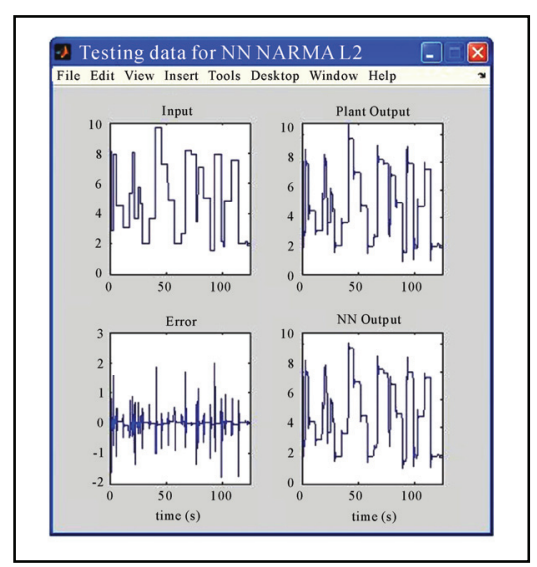

Figure 4. Testing the signals for the Narma L2 Controller. 


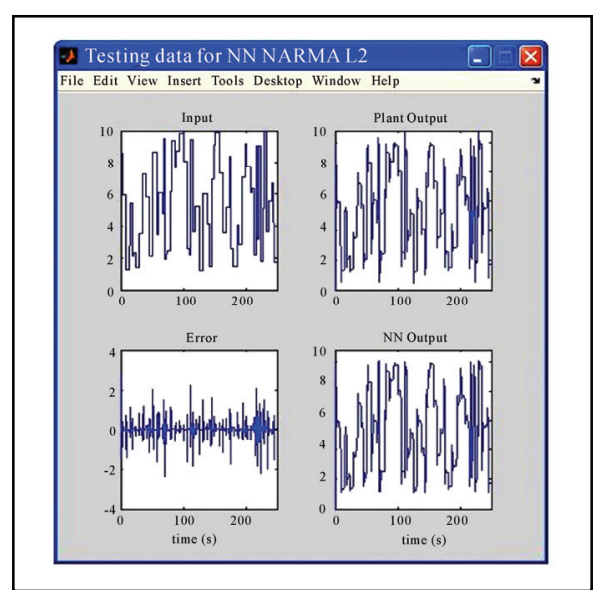

Figure 5. Training the signals for the Narma L2 Controller.

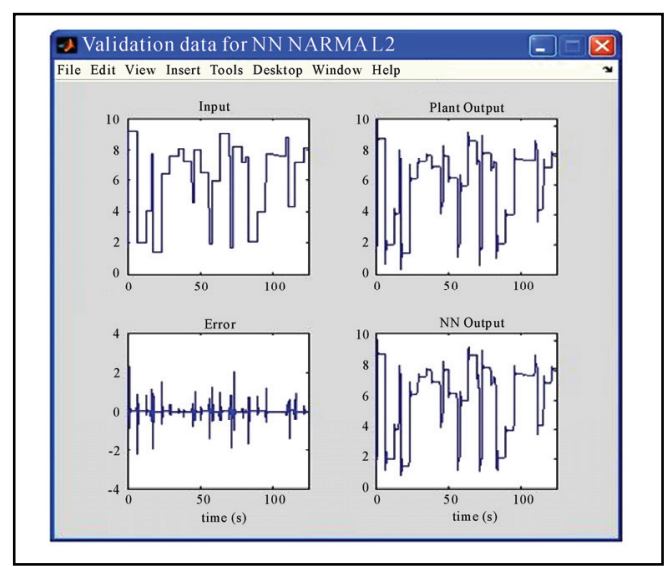

Figure 6. Validation of signals of Narma L2 Controller.

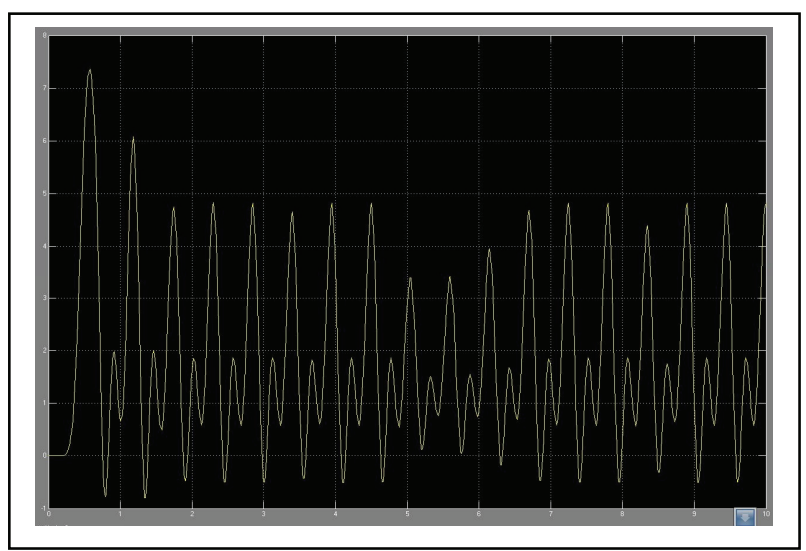

Figure 7. The final graphical result of the signal versus time of the bionic arm. identify the upper limb action signals after this training process has been completed.

\section{CONCLUSIONS}

The aim of this paper was to give a theoretical analysis of a concept which can be implemented in practice. This would also help to concentrate about the limitations of the artificial upper limbs which can only perform a very few actions making amputees all the more conscious of their developed deficiencies.

\section{ACKNOWLEDGMENT}

I would like to take this opportunity to thank Dr. Meghamala Dutta and Dr. Avijit Talukdar who had provided insightful knowledge regarding the hypothetical analysis of bionic arm.

\section{REFERENCES}

[1] Joseph, D.B. (2004) Handbook of biomedical engineering. CRC Press.

[2] George, B., Gwilym, M.J. and Gregory, C.R. (1994) Time series analysis: Forecasting and control, 3rd Edition, Prentice-Hall.

[3] Mills, T.C. (1990) Time series techniques for economists. Cambridge University Press, Cambridge.

[4] Pandit, Sudhakar M. and Wu, S.M. (1983) Time series and system analysis with applications. John Wiley\& Sons, Inc.

[5] Mathworks-MatLab Software. 